C 1996 IEEE. Personal use of this material is permitted. However, permission to reprint/republish this material

for advertising or promotional purposes or for creating new collective works for resale or redistribution to servers

or lists, or to reuse any copyrighted component of this work in other works must be obtained from the IEEE.

\title{
Optimizing the Luminosity in the Tevatron by Independently Moving the Horizontal and Vertical Beta Stars Longitudinally
}

\author{
M. A. Martens, G. P. Goderre \\ Fermi National Accelerator Laboratory \\ P. O. Box 500, Batavia, IL 60510, USA
}

\begin{abstract}
The low beta tripplet in the Tevatron is instrumented such that it is possible to move the horizontal and/or the vertical beta star in the longitudinal direction. This control over beta star allows us to minimize the longitudinal separation of horizontal and vertical beta star at each interaction region independently, thus maximizing the luminosity. Results of varying the longitudinal separation of horizontal and vertical beta star are shown and the sensitivity of the luminosity on this parameter is discussed.
\end{abstract}

\section{Introduction}

In the Fermilab Tevatron a series of low beta quadrupoles are used to transversely focus the proton and antiproton bunches as they collide in the B0 (CDF) and D0 interaction regions. The Tevatron lattice is designed such that the center of the experimental detectors is aligned longitudinally with both the minimum of $\beta_{x}$ and the minimum of $\beta_{y}$. Due to imperfections in the lattice however, the minimum $\beta_{x}$ and minimum $\beta_{y}$ may not coincide resulting in a lower luminosity and a shift in the longitudinal luminosity distribution. The luminosity may also be reduced if the centers of the proton and antiproton bunches do not collide at the same place as the minimum $\beta$. Therefore the luminosity is maximized by aligning the collision point and minimum $\beta$.

Changes to the collision point in the Tevatron are easily made by cogging the antiproton bunches with respect to the proton bunches. As we will show in this paper, the positions of the minimum $\beta$ can also be changed by adjusting the currents in the low beta quadrupoles near the interaction regions. Using a combination of the $\beta$ moves and cogging changes we have been able to increase the luminosity at CDF and D0 and to better center the longitudinal luminosity distribution in the CDF detector.

In the next section we introduce the $\alpha$-bumps which are used to change the position of the minimum $\beta$. We then give some examples of the sensitivity of the luminosity on mis-alignments of the minimum $\beta$ and collision point. Finally we give some results of implementing these $\alpha$-bumps in the Tevatron.

\section{II. $\alpha$-bump}

Figure 1 shows a sketch of the low beta quadrupoles on either side of one of the interaction regions in the Tevatron. For our purposes the currents in these magnets can be thought of as being supplied by four power supplies: 1 on Q3U, 1 on

Operated by the Universities Research Association,Inc, under contract with the U.S. Department of Energy

Q3D, 1 on Q2U and Q4U in series, and 1 on Q2D and Q4D in series.* By changing the currents in these magnets in the proper ratio it is possible to change the value of $\alpha=-\frac{1}{2} \frac{d \beta}{d s}$ in the center of the interaction region. The net effect of this $\alpha$-bump is to move the position of the minimum $\beta$ without changing the value of the minimum $\beta$ and without changing significantly the lattice outside of the tripplet magnets.

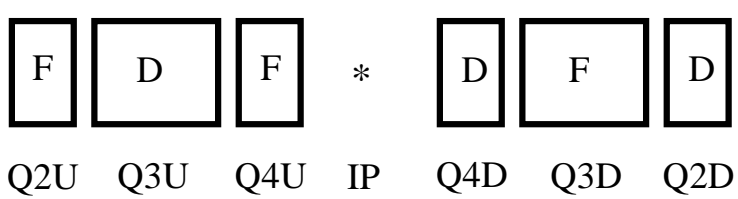

Figure. 1. Sketch of the positions of the low beta tripplet quads around the interaction point (IP). F (D) are focusing (defocusing) in the horizontal plane.

The simplest way to understand how the $\alpha$-bump works is by first realizing that phase advance $\Delta \psi$ between the upstream set of magnets and the downstream set is nearly 180 degrees. This makes it possible to start a $\beta$-wave in the upstream magnets and almost identically cancel the $\beta$-wave in the downstream magnets. To determine the ratio of current changes in the quadrupole magnets for a horizontal $\alpha$-bump, for instance, we use the constraints: 1) start a $\beta_{x}$-wave in the upstream magnets, 2) cancel the $\beta_{x}$-wave in the downstream magnets, 3 ) create no $\beta_{y}$-wave in the upstream magnets, and 4) create no $\beta_{y}$-wave in the downstream magnets.

The $\beta$-wave created by a quadrupole kick of strength $q=$ $B^{\prime} l /(B \rho)$ at a location where the $\beta$ has a value of $\beta_{1}$ is given by [2]

$$
\frac{\Delta \beta}{\beta}=-q \beta_{1} \sin 2 \psi_{0}+\frac{1}{2}\left(q \beta_{1}\right)^{2}\left(1-\cos 2 \psi_{0}\right)
$$

where $\psi_{0}$ is the phase advance of the unperturbed $\beta$ function. Since the value of $\beta$ is large in the quadrupole magnets the phase advance through the tripplet magnets is very small $(0.4$ degrees) and we can make the approximation that phase advance between the magnets and the center of the interaction region is 90 degrees. This simplifies the calculation of the $\alpha$-bump since we can treat the quadrupole kicks from the 3 upstream magnets

\footnotetext{
*In reality the Q2 and Q4 magnets are on one buss with a trim supply to add current to the upstream magnets and the Q3 magnets are on one buss with a trim supply to add current to the upstream magnet.
} 
as single kick with strength

$$
\kappa_{x}=-q_{\mathrm{Q} 2 \mathrm{U}} \beta_{x, \mathrm{Q} 2 \mathrm{U}}+q_{\mathrm{Q} 3 \mathrm{U}} \beta_{x, \mathrm{Q} 3 \mathrm{U}}-q_{\mathrm{Q} 4 \mathrm{U}} \beta_{x, \mathrm{Q} 4 \mathrm{U}}
$$

where the $\beta_{x, \mathrm{Q} 2 \mathrm{U}}$, for instance, is the average beta function in the Q2U quadrupole, and $q_{\mathrm{Q} 2 \mathrm{U}}$ is the change in gradient strength multiplied by the length of the magnet. The resulting $\beta$-wave is then given by

$$
\frac{\Delta \beta_{x}}{\beta_{x}}=\kappa_{x} \sin 2 \psi_{0}+\frac{1}{2} \kappa_{x}^{2}\left(1-\cos 2 \psi_{0}\right)
$$

Ignoring any coupling effects, and assuming the unperturbed $\beta$ function in the interaction region is given by

$$
\beta(z)=\beta^{*}+\frac{z^{2}}{\beta^{*}}
$$

we can use Eq. 3 to calculate the new $\beta$ function with the $\alpha$-bump in place,

$$
\beta_{x, \text { new }}=\beta_{x}^{*}+\frac{\left(z+\kappa_{x} \beta_{x}^{*}\right)^{2}}{\beta_{x}^{*}} .
$$

As Eq. 5 shows, there is no change in the value of the minimum $\beta$ and the position of the minimum $\beta$ is moved by $-\kappa_{x} \beta_{x}^{*}$. A similar expression holds in the vertical plane as well. By choosing $\kappa_{x}$ and $\kappa_{y}$ in the correct ratio we can change the horizontal beta function and leave the vertical beta function unchanged.

During colliding beam operations the collision point and $\alpha$ bump strengths are adjusted to maximize the luminosities at B0 and D0 separately.

\section{Luminosity dependence on $\alpha$-bumps}

The luminosity of proton and antiproton bunches colliding in the Fermilab Tevatron is given by the luminosity overlap integral [1]

$$
\checkmark=\frac{f_{\mathrm{rev}} N_{p} N_{\bar{p}}}{\sqrt{2 \pi}^{3} \sigma_{z}} \int \frac{\exp \left(-\left(z-z_{0}\right)^{2} / 2 \sigma_{z}^{2}\right)}{\sigma_{x}(z) \sigma_{y}(z)} d z .
$$

In this expression it is assumed that the longitudinal and transverse beam profiles are Gaussian and we ignore coupling and differences in the proton and antiproton orbits. As Eq. 6 shows, the luminosity is affected by such things as the collision point $z_{0}$, the longitudinal widths of the bunches $\sigma_{z}^{2}=\left(\sigma_{z, p}^{2}+\sigma_{z, \bar{p}}^{2}\right) / 4$, and the transverse width of the bunches $\sigma_{x}^{2}(z)=\sigma_{x, p}^{2}(z)+\sigma_{x, \bar{p}}^{2}(z)$. The widths of the particle bunches are a function of the emittances, momentum spread and $\beta$ function, $\sigma_{x}^{2}=\epsilon_{x} \beta_{x}^{*}+\left(\eta_{x} \sigma_{\delta}\right)^{2}$. Therefore the luminosity is also dependent on the $\beta$ functions.

To demonstrate the effects that offsets in the collision point and position of the minimum beta have on luminosity we use Eq. 6 with a set of typical beam parameters for collider operations. The $95 \%$ normalized emittances we use are $\epsilon_{x, p}=$ $\epsilon_{y, p}=25 \pi, \epsilon_{x, \bar{p}}=\epsilon_{y, \bar{p}}=15 \pi$, and $\sigma_{z}=45 \mathrm{~cm}$. Fig. 2, for instance, shows the reduction in luminosity as a function of an offset in the $\beta_{x}$. As seen in Fig. 2 the luminosity is $5 \%$ below optimum when the minimum $\beta_{x}$ is moved by $20 \mathrm{~cm}$. Offsets in the collision point have a similar effect on the luminosity as shown in Fig. 3.

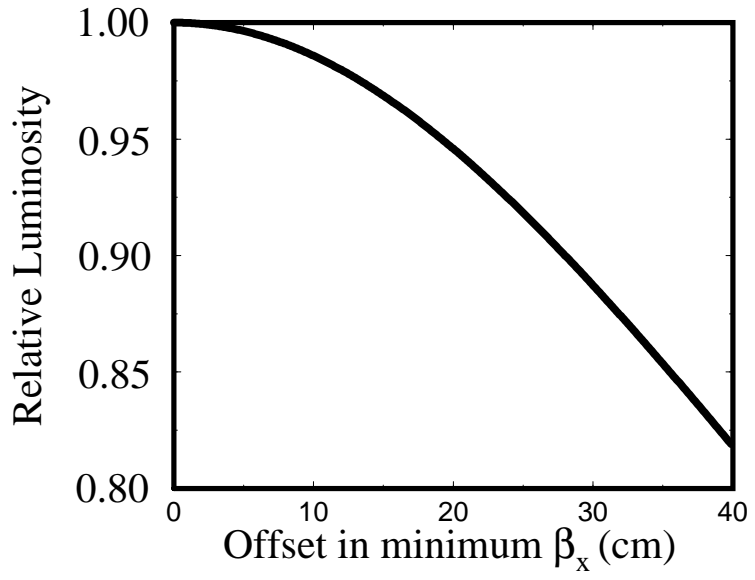

Figure. 2. Luminosity as a function of offset in the minimum $\beta_{x}$ for typical collider operations.

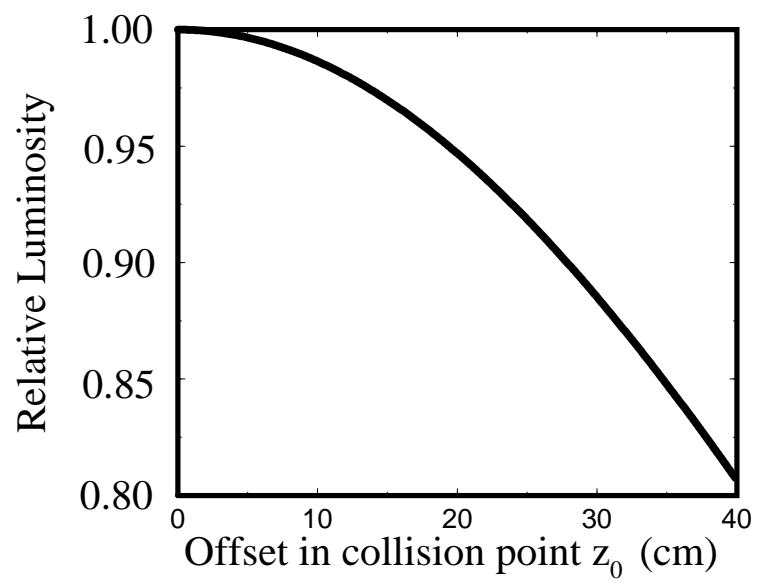

Figure. 3. Luminosity as a function of collision point offset $z_{0}$ for typical collider operations.

In addition to lowering the luminosity offsets in the minimum $\beta$ and collision point also affect the longitudinal distribution of the luminosity $d^{\triangleleft} / d z$. Fig. 4 shows the effect of a $20 \mathrm{~cm}$ offset in the position of $\beta_{x}$. The dashed line is the luminosity distribution for the nominal parameters and the solid line is the luminosity distribution with the offset $\beta_{x}$. Both the reduction in luminosity as well as the shift in the distribution are apparent.

\section{Conclusions}

The $\alpha$-bumps described above were implemented and used in the Tevatron during collider run IB. An example of a $\alpha$ bump scan is shown in Fig. 5. In this particlular example the luminosity at D0 was recorded as the magnitude of the horizontal $\alpha$-bump was changed.

After several iterations of $\alpha$-bump scans and cogging scans we found that we could increase the luminosity at D0 by $3 \%$, increase the luminosity at CDF by $1 \%$ and move the center of the distribution at CDF from $+4.5 \mathrm{~cm}$ to within $1 \mathrm{~cm}$ of the center of the detector. The net changes to the cogging and positions of the minimum $\beta$ are shown in table I. 


\section{References}

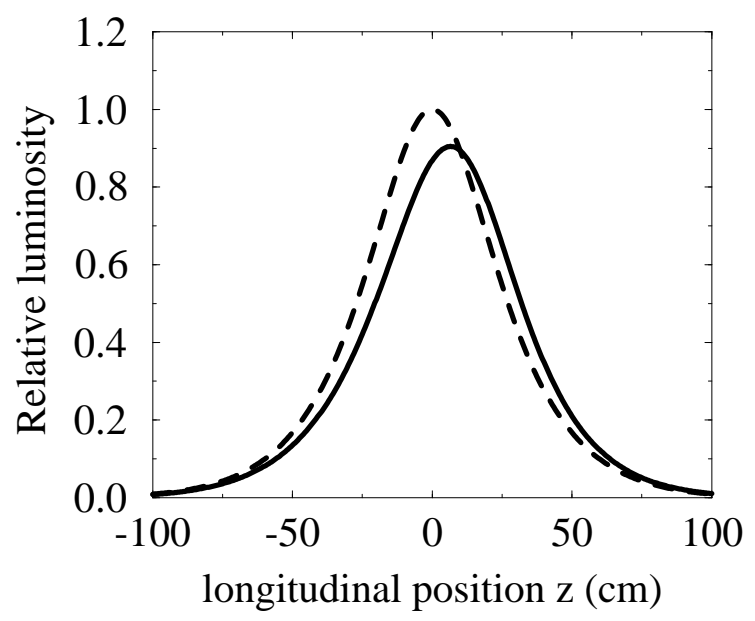

Figure. 4. Change in the luminosity distribution created by an $+20 \mathrm{~cm}$ offset in the position on minimum $\beta_{x}$. The dashed line is the nominal distribution and the solid is the distribution with the offset.

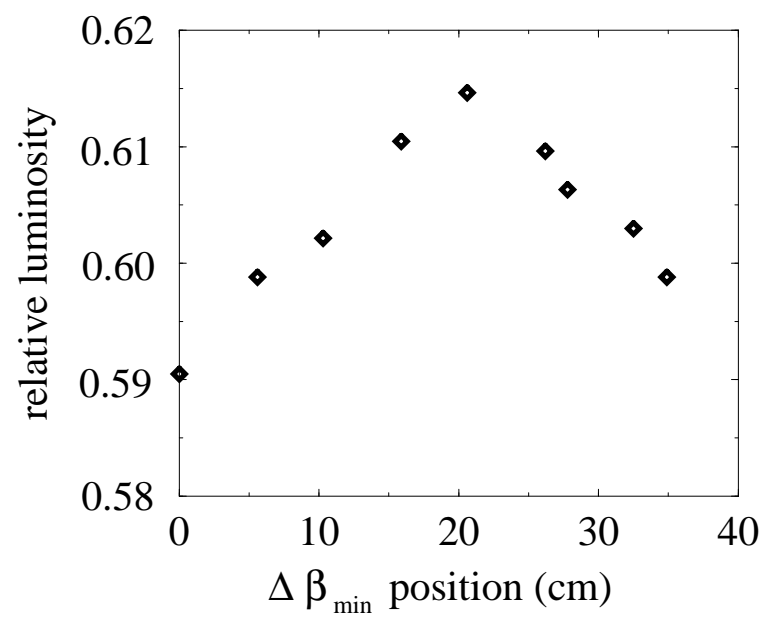

Figure. 5. Measured luminosity at D0 as a function of $\alpha_{x}$ bump amplitude. This measurement shows that moving the $\beta_{x}$ minimum by $+20 \mathrm{~cm}$ would maximize luminosity.

\begin{tabular}{|l|r|}
\hline$\Delta z_{0}$ & $-6 \mathrm{~cm}$ \\
$\Delta \beta_{x}$ at $\mathrm{CDF}$ & $-11 \mathrm{~cm}$ \\
$\Delta \beta_{y}$ at $\mathrm{CDF}$ & $0 \mathrm{~cm}$ \\
$\Delta \beta_{x}$ at $\mathrm{D} 0$ & $-4 \mathrm{~cm}$ \\
$\Delta \beta_{y}$ at $\mathrm{D} 0$ & $+13 \mathrm{~cm}$ \\
\hline
\end{tabular}

Table I

Net changes made to Tevatron lattice as a result of cogging and $\alpha$-bump scans.
[1] M. Month, "Collider Performance with Ideal Collisions", "Accelerator Division Report 85-1", (1985)

[2] D.A. Edwards and M.J. Syphers, "An Introduction to the Physics of High Energy Accelerators", John Wiley and Sons, Inc., New York, (1993) 